Meta

Journal des tradlucteurs

Translators' Journal

\title{
Un nouveau type de dictionnaire utile aux traducteurs : le DEC du français moderne
}

\section{Betty Cohen et Léo Elnitsky}

Volume 29, numéro 2, juin 1984

URI : https://id.erudit.org/iderudit/002346ar

DOI : https://doi.org/10.7202/002346ar

Aller au sommaire du numéro

Éditeur(s)

Les Presses de l'Université de Montréal

ISSN

0026-0452 (imprimé)

1492-1421 (numérique)

Découvrir la revue

Citer cet article

Cohen, B. \& Elnitsky, L. (1984). Un nouveau type de dictionnaire utile aux

traducteurs : le DEC du français moderne. Meta, 29(2), 159-174.

https://doi.org/10.7202/002346ar d'utilisation que vous pouvez consulter en ligne.

https://apropos.erudit.org/fr/usagers/politique-dutilisation/ 


\title{
ÉTUDES \\ TERMINOLOGIQUES \\ ET LINGUISTIQUES
}

\author{
UN NOUVEAU TYPE DE DICTIONNAIRE UTILE AUX TRADUCTEURS : \\ LE DEC DU FRANÇAIS MODERNE \\ (articles PRIX dans le DEC)*
}

Ce sont les traducteurs qui utilisent le plus les dictionnaires et ce sont eux aussi qui en sont le moins satisfaits. Leur insatisfaction vient en grande partie du fait que, traditionnellement, presque tous les dictionnaires mettent l'accent en premier lieu sur la représentation du sens des mots qu'ils retiennent, en leur opposant soit une définition, dans le cas d'un dictionnaire unilingue, soit une traduction, dans le cas d'un dictionnaire bilingue. La définition ou la traduction peuvent être accompagnées d'indications morphologiques et d'informations syntaxiques succinctes d'ordre très général, telles que, par exemple, transitif ou intransitif pour un verbe, mais dans la plupart des cas c'est tout ce qu'on y trouve. Ainsi les dictionnaires ne sont utiles aux traducteurs que dans la première étape de leur travail - analyse du texte à traduire et recherche de lexèmes équivalents dans la langue d'arrivée.

L'usage du dictionnaire s'arrête là, et il est vrai que dans beaucoup de cas cela suffit pour rédiger le texte cible - puisque, après tout, la plupart des traducteurs traduisent dans leur langue maternelle. Mais même quand on travaille dans sa langue maternelle on se heurte à un bon nombre de problèmes linguistiques assez délicats parce que, d'une part, tout le monde ne maîtrise pas sa langue maternelle au même degré et, d'autre part, cela étant encore plus important, le plus souvent ce n'est pas dans la langue commune qu'est faite la traduction mais dans des sous-langages spécifiques (scientifique, technique, juridique, économique, etc.) que le traducteur ne connaît qu'imparfaitement.

C'est pourquoi, lorsqu'on rédige le texte cible, on a souvent besoin d'informations de deux types :

1) le régime syntaxique d'un mot et les façons concrètes de l'associer à ses compléments (la cooccurrence syntaxique) : par exemple, escompter les prestations de $25 \%$;

2) la capacité d'un mot de former des locutions figées avec d'autres mots qui servent à exprimer, en fonction du mot donné, des sens ou des relations sémantiques spécifiques (la cooccurrence lexicale restreinte ; il s'agit ici des sens tels que 'le haut/bas dégré' avec un mot désignant une caractéristique, ou 'faire', 'commencer', 'finir' avec des mots désignant une action, etc.).

\footnotetext{
* Le présent travail a été effectué dans le cadre du projet "Études lexico-sémantiques du français contemporain selon le modèle "Sens-Texte " au Département de linguistique et philologie de l'Université de Montréal (projet de recherche subventionné par le Conseil de recherches en sciences humaines du Canada ; subvention $n^{\circ}$ 410-82-0549).
} 
Les informations du premier type ne sont données de façon explicite et complète dans aucun dictionnaire. (Notons cependant qu'il existe deux dictionnaires unilingues anglais, destinés à ceux qui apprennent cette langue - Oxford Advanced Learner's Dictionary of Current English par A.S. Hornby et Longman Dictionary of Contemporary English - où l'on retrouve plus qu'ailleurs ces informations. Mais même là elles restent trop générales ne s'appliquant qu'aux groupes de mots et non aux mots individuels.)

Les informations du deuxième type (encore plus importantes pour le traducteur) peuvent être trouvées en petit nombre dans les dictionnaires généraux et parfois dans quelques dictionnaires techniques. C'est seulement dans les plus grands dictionnaires, tels que le Grand Robert qu'il y en a beaucoup - ordinairement dans les exemples contenant des locutions figées avec le lexème clé. Il existe aussi un dictionnaire anglais destiné spécialement à fournir des mots souvent utilisés avec un mot donné (adverbes pour un verbe ou un adjectif, adjectifs et verbes pour un nom, etc.) : The Word Finder (Rodale, 1976), et il est remarquable qu'il s'adresse en premier lieu aux locuteurs natifs et non uniquement à ceux dont la langue maternelle est autre que l'anglais. Mais les grands dictionnaires, ainsi que The Word Finder, ont le même trait commun : l'ensemble de locutions figées, qui y est présenté, n'est jamais exhaustif ; les locutions données le sont en vrac, aucune théorie linguistique n'ayant été utilisée pour les classer.

C'est pourquoi nous croyons que les lecteurs de Meta s'intéresseront à une description lexicographique (le mot français PRIX) faite pour un dictionnaire d'un type tout à fait nouveau qui se distingue des dictionnaires des générations précédentes par trois aspects importants du point de vue de ce que nous avons dit ci-dessus :

1) ce dictionnaire est basé sur un ensemble cohérent d'idées linguistiques et lexicographiques tout à fait originales (voir Mel'čuk 1978, 1981 ; Mel'čuk et al., 1981);

2) on vise à décrire tous les lexèmes que le dictionnaire contient de façon aussi complète que possible avec l'accent sur la cooccurrence syntaxique et lexicale ;

3) les descriptions sont systématiques, cohérentes et rigoureusement formelles.

Le dictionnaire en question - Dictionnaire explicatif et combinatoire du français contemporain (DEC) - est actuellement en cours d'élaboration au Département de linguistique et philologie de l'Université de Montréal. C'est un ouvrage d'un caractère plutôt scientifique, comme le sont d'ailleurs presque tous les dictionnaires à plusieurs volumes, mais qui peut, néanmoins, être très utile, toujours comme ceux-ci, au grand public et aux traducteurs. Il peut aussi, nous l'espérons, servir de modèle pour de nouveaux dictionnaires techniques destinés spécialement aux traducteurs.

Le premier volume d'une série s'intitulant Dictionnaire explicatif et combinatoire du français contemporain, Recherches lexico-sémantiques doit paraitre aux Presses de l'Université de Montréal en août 1984. Ce volume contient quatre exposés théoriques, fournissant une description très détaillée du DEC, ainsi qu'environ 200 articles de dictionnaire.

Les articles du mot PRIX que nous présentons ici (et qui ne figurent pas dans le volume précité) donneront aux lecteurs de Meta un avant-goût de ce premier fragment du DEC. N'étant pas en mesure de clarifier dans cette introduction tous les détails théoriques et formels, nous renvoyons le lecteur à une autre publication - Mel'čuk et al. (1981) - qui renferme nombre de renseignements sur le DEC. Néanmoins, nous voulons donner ici-même quelques explications pour que le lecteur puisse mieux comprendre ce qui suit même sans recourir à d'autres sources d'information.

L'article du DEC est divisé, en gros, en trois zones :

1) Une zone sémantique - la définition qui décrit le sens du lexème de façon complète et formelle. Si le lexème désigne une situation impliquant plusieurs participants 
nommés de façon générale dans la définition et concrétisés auprès du lexème dans le rôle de ses dépendants syntaxiques (compléments), ces participants sont marqués par des variables $(X, Y, Z, \ldots)$. De cette manière on représente les actants sémantiques du lexème auxquels correspondent au niveau du texte (niveau de surface) ses actants syntaxiques (ses compléments).

2) Une zone syntaxique - le schéma de régime qui décrit la cooccurrence syntaxique du lexème en spécifiant les façons différentes de réaliser ses actants syntaxiques et de les y joindre.

3) Une zone de cooccurrence lexicale restreinte - les fonctions lexicales. Nous nous étendrons davantage sur cette troisième zone, car elle présente plus d'intérêt pour nos lecteurs. La zone est divisée en deux colonnes. En général, les deux colonnes opposent deux choses : une partie très importante de ce qu'on dit dans la langue relativement au lexème clé et la façon dont on le dit. La colonne de gauche comporte une liste de sens, assez abstraits dans l'ensemble, qui se conforment à l'une des deux conditions suivantes :

- un sens donné à gauche doit se rapporter à un élément suffisamment important (non trivial) du sens du lexème clé ;

- un sens donné à gauche est exprimé dans la langue de façon phraséologique, c'est-à-dire de façon irrégulière, spécifique pour le lexème clé et en fonction de ce dernier. Dans la plupart des cas, les deux conditions sont satisfaites simultanément, ce qui est tout à fait naturel, car les sens les plus importants pour un lexème, qui apparaissent avec lui le plus souvent, sont en règle générale exprimés de façon idiomatique. Des sens de ce type sont appelés "fonctions lexicales", justement à cause du rapport spécifique entre eux et leurs réalisations différentes auprès des lexèmes différents (voir Mel'čuk et al., 1981 ; Mel’čuk 1982 ; Iordanskaja et Arbatchewsky-Jumarie, 1982).

La colonne de droite présente les lexèmes ou syntagmes qui expriment les sens donnés à gauche en fonction du lexème clé. Ceux-ci constituent un ensemble d'unités lexicales qui non seulement sont utilisées le plus souvent avec le lexème clé, mais qui également, et ceci est plus important encore, l'individualisent en formant son " cortège " lexical spécifique. En général, ce qu'on donne dans la zone de cooccurrence lexicale restreinte fait partie des informations portant sur les particularités du lexème clé qu'il faut connaitre spécialement pour utiliser ce lexème de façon correcte et idiomatique. Le reste de la cooccurrence est libre et n'est restreint que par les conditions communes à tous les termes de la même classe syntaxique et/ou sémantique.

Il a été établi (voir Žolkovskij et Mel'čuk, 1967) qu'un certain nombre de sens (= fonctions lexicales), se conformant aux conditions mentionnées ci-dessus, sont exprimés très souvent par rapport à des mots très divers. Ces sens "standards " sont connus comme les fonctions lexicales standards, et un système de symboles, simples et combinés, est utilisé pour les désigner. Des sens plus spécifiques qui ne peuvent pas être décrits en termes de symboles des FL standards (sens " non standard ") sont définis à l'aide des mots de la langue de description. On utilise aussi des combinaisons de symboles des FL avec des mots de la langue de description.

Étant donné que les fonctions lexicales sont présentées dans un certain ordre, l'utilisateur du DEC qui s'est familiarisé avec le système peut facilement trouver le sens et l'unité lexicale correspondante dont il a besoin. L'usage des FL demande, bien sûr, une certaine pratique, mais il n'est pas du tout nécessaire d'être linguiste pour s'en servir. Quiconque travaille plus ou moins régulièrement avec des langues (traducteur, pro- 
fesseur, etc.) peut maîtriser facilement le système des FL $L^{1}$. Il est à noter qu'on peut utiliser le DEC sans connaître les FL - dans l'un ou l'autre cas l'utilisateur y trouvera une liste exhaustive ${ }^{2}$ des unités lexicales idiomatiques qui se combinent avec le lexème clé.

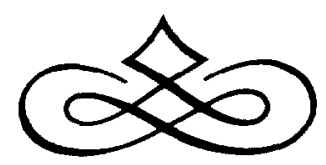

PRIX, nom masc.

I.1. Valeur de $\mathrm{X}$ pour $\mathrm{Y}$ [Le prix de l'amitie]

2a. Quantité de monnaie $Y$ censée représenter la valeur d'une marchandise $X$ [Le prix d'une marchandise]

2b. Étiquette indiquant le prix 1.2a [Coller le prix]

3. $\mathrm{X}$ dont $\mathrm{Z}$ paye III.1 $\mathrm{Y}$ [Le prix du succès]

4. $\mathrm{X}$ dont $\mathrm{Z}$ paye III.2 $\mathrm{Y}[$ Le prix $d u$ crime]

5. $\mathrm{X}$ dont $\mathrm{Z}$ paye II.a $\mathrm{Y}$ [Le prix $d u$ dévouement]

II.1. Récompense offerte dans le cadre d'un concours [le prix de $1000 \$$ pour le meilleur roman]

2a. Oeuvre pour laquelle l'auteur a reçu un prix II.1 [ $T u$ dois lire le dernier prix Goncourt]

2b. Personne qui a reçu un prix II.1 [Elle a épousé un prix de musique]

1. Une liste des FL peut être trouvée dans Mel'čuk et al. 1981.

2. En ce qui concerne l'exhaustivité des informations données dans la zone de la cooccurrence lexicale, il faut souligner que l'article du mot PRIX présenté ici a été écrit pour un DEC général, dans lequel n'est consigné que l'usage courant à l'exclusion d'usages très spécialisés. Il est entendu qu'un dictionnaire spécialisé (commercial ou économique) devrait donner nombre d'expressions supplémentaires exclusivement utilisées par des spécialistes. 
I.1. pas de pl. Prix de $X$ pour $Y=$ Valeur $1^{*}$ de $X$ pour $Y$

Régime

\begin{tabular}{|c|l|}
\hline $1=\mathrm{X}$ & \multicolumn{1}{|c|}{$2=\mathrm{Y}$} \\
\hline 1. de $\mathrm{N}$ & 1 . pour $\mathrm{N}$ \\
\hline
\end{tabular}

$\mathrm{C}_{1} \quad$ : le prix de l'amitié 〈de nos amis〉

$\mathrm{C}_{1}+\mathrm{C}_{2} \quad$ : le prix de la richesse pour les hommes

\section{Fonctions lexicales}

Syn

Magn $+\mathbf{A}_{1}$

: valeur 1

Magn

: sans prix

Oper 1

: grand, haut

: avoir [du ]//valoir ART/A poss prix [Chacun vaut son prix]

Magn + Oper $_{1}$

: // n'avoir pas de prix

IncepOper $_{1}$

$:$ gagner, prendre [du $\sim]$ [Une chose gagne $\langle$ prend $\rangle d u$ prix dès qu'elle devient rare]

Plus $_{1}+$ IncepOper $_{1}$

: gagner [du ] [Ce tableau gagne du prix à mesure

Minus $_{1}+$ FinOper $_{1}$ qu'on le regarde]

$\mathrm{Oper}_{2}$

CausFunc $_{1}$

$:$ perdre $[\mathrm{du} \sim]$

Labor $_{21}$

$:$ attacher $[\mathrm{du} \sim$ à $\mathrm{N}]$

$:$ donner $[\mathrm{du} \sim$ à $\mathrm{N}]$; mettre $[\mathrm{le} \sim$ à $\mathrm{N}]$; vieilli donner $[\sim$ à $\mathrm{N}]$ [La rareté du fait donnait prix à la chose] $:$ mettre $[\mathrm{N}$ à ART $\sim] \mid$ P. $\stackrel{\text { ATTR }}{\longrightarrow}$ Magn

\section{Exemples}

On ne comprend généralement le prix d'une bonne réputation qu'après l'avoir perdue [Gide]. Elle était liante et familière, et son esprit donnait du prix à cette familiarité [Rousseau]. Notre humeur met le prix à tout ce qui nous vient de la fortune [La Rochefoucault]. Le prix que nous valons, qui le sait mieux que nous [Corneille]. Aucun

* Valeur 1 de $X$ pour $Y=$ Importance que $\mathrm{Y}$ attribue à $\mathrm{X}$, de par les qualités physiques, intellectuelles ou morales de $X$. 
sentiment n'a plus de prix que cette confiance d'un homme dans un autre homme, car ce sentiment est la somme de tous les autres. Je mets son estime au plus haut prix. J'attache beaucoup de prix à son amitié. Cet homme est sans prix, il est d'un rare mérite.

1.2a. Prix pour $X$ de $Y$ [de $Z$ de $W]^{3}=$ Quantité de monnaie (ou l'équivalent) $Y$ censée représenter la valeur $2^{*}$ d'une marchandise $X$ ou d'un service $X$ que le vendeur $Z$ de $X$ veut que l'acheteur $W$ de $X$ lui paie I.1a.**

\section{Régime}

\begin{tabular}{|l|l|l|l|}
\hline \multicolumn{1}{|c|}{$1=\mathrm{X}$} & \multicolumn{1}{|c|}{$2=\mathrm{Y}$} & $3=\mathrm{Z}$ & $4=\mathrm{W}$ \\
\hline $\begin{array}{l}\text { 1. de } \mathrm{N} \\
\text { 2. pour } \mathrm{N}\end{array}$ & 1. de $\mathrm{N}_{\text {num }}$ & - & - \\
\hline
\end{tabular}

1) $\mathrm{C}_{1}+\mathrm{C}_{2}$

: non souhaitable

$\mathrm{C}_{1}$

le prix d'une robe / pour une robe

$\mathrm{C}_{2}$

: le prix de 30 dollars

Non souhaitable

: ?le prix de $100 \$$ de cette marchandise... (1)

$[=$ le prix de cette marchandise qui est de 100 \$...]

\section{Fonctions lexicales}

$\begin{array}{ll}\text { Syn } & : \text { valeur } 2 \\ \text { Syn }_{\subset} & : \text { coût } 1 \\ \mathbf{S}_{1} & : \text { marchandise } \\ \mathbf{S}_{2} & : \text { montant [de ART } \sim] \\ \text { Magn }+\mathbf{A}_{1} & : \text { de } \sim \text { [un bijou de prix] }<\text { sans } \sim\end{array}$

3. La structure des actants sémantiques et syntaxiques du lexème PRIX I.2a est celle adoptée dans le DEC pour tous les lexèmes paramétriques (vitesse, poids, age, etc.). Une discussion détaillée de la description de tels lexèmes est donnée dans Elnitsky et Mel'cuk (à paraître).

* Valeur 2 de $X=$ Valeur 1 de $\mathrm{X}$ telle qu'on désire $\mathrm{X}$ et qu'on est prêt à payer I. la pour obtenir X.

** $X$ paye I. Ia $Y$ en $Z \grave{a} W$ pour $U=\mathrm{X}$ remet une somme d'argent $\mathrm{Y}$ sous une forme $\mathrm{Z}$ à $\mathrm{W}$ en échange d'un bien ou d'un service $U$. 
Magn

\section{AntiMagn}

trop AntiMagn

à la suite d'une faveur que $Z$

fait à W, AntiMagn : d'ami, de faveur, préférentiel

par rapport aux prix pratiqués

par les autres vendeurs,

AntiMagn : concurrentiel, compétitif

tel que $\mathrm{Z}$ subit une perte,

$$
\text { AntiMagn : sacrifié }
$$

établi dans un cas particulier

(normalement dans le but d'ac-

célérer la vente de $\mathrm{X}$ ),

AntiMagn : spécial

établi dans le but d'accélérer la

vente de $X$ dont $Z$ veut se dé-

barasser le plus vite possible,

AntiMagn : de liquidation, d'écoulement, de solde ; démarqué, dégriffé, bradé, gâché

établi dans le but d'accélérer la

vente de $\mathrm{X}$ à la fin de la saison,

établi dans le but d'attirer $W$

AntiMagn : de fin de saison

dans le magasin, AntiMagn : d'appel, de vente publicitaire

établi dans le but de pénétrer

dans le marché d'un autre pays,

inférieur au P. du marché natio-

nal et souvent au $P$. de revient,

AntiMagn : de bataille, de dumping

tel que $\mathrm{Z}$ pratique quand $\mathrm{W}$

paie pour $X$ immédiatement et

en argent liquide, AntiMagn : au comptant

IncepPredPlus 2

$\mathrm{S}_{0}$ IncepPredPlus 2

$F_{1}=$ brusquement

: augmenter 1, monter 1, grimper ; spéc. s'alourdir

: augmentation 1 , montée 1 , hausse, ascension [de ART ]

IncepPredPlus 2 : décupler, bondir

$\mathrm{S}_{0} \mathrm{~F}_{1} \quad:$ bond, sursaut, poussée [de ART $\sim$ ]

$F_{2}=$ brusquement

MagnIncepPredPlus 2 : s'envoler, s'emballer, flamber, monter 1 en flèche

$\mathrm{S}_{0} \mathrm{~F}_{2}$

envolée, flambée [de ART ]

Caus $_{(3)}$ IncepPredPlus 2

hausser, augmenter 2, monter 2, relever, majorer

[ART $\sim$ de Num + N]

Caus $_{(3)}$ MagnIncepPredPlus $_{2} \quad:$ gonfler [ART $\sim$ ]

$\mathrm{F}_{3}=$ nonPermIncepPredPlus $2 \quad:$ geler, bloquer $[\mathrm{ART} \sim$ ] 


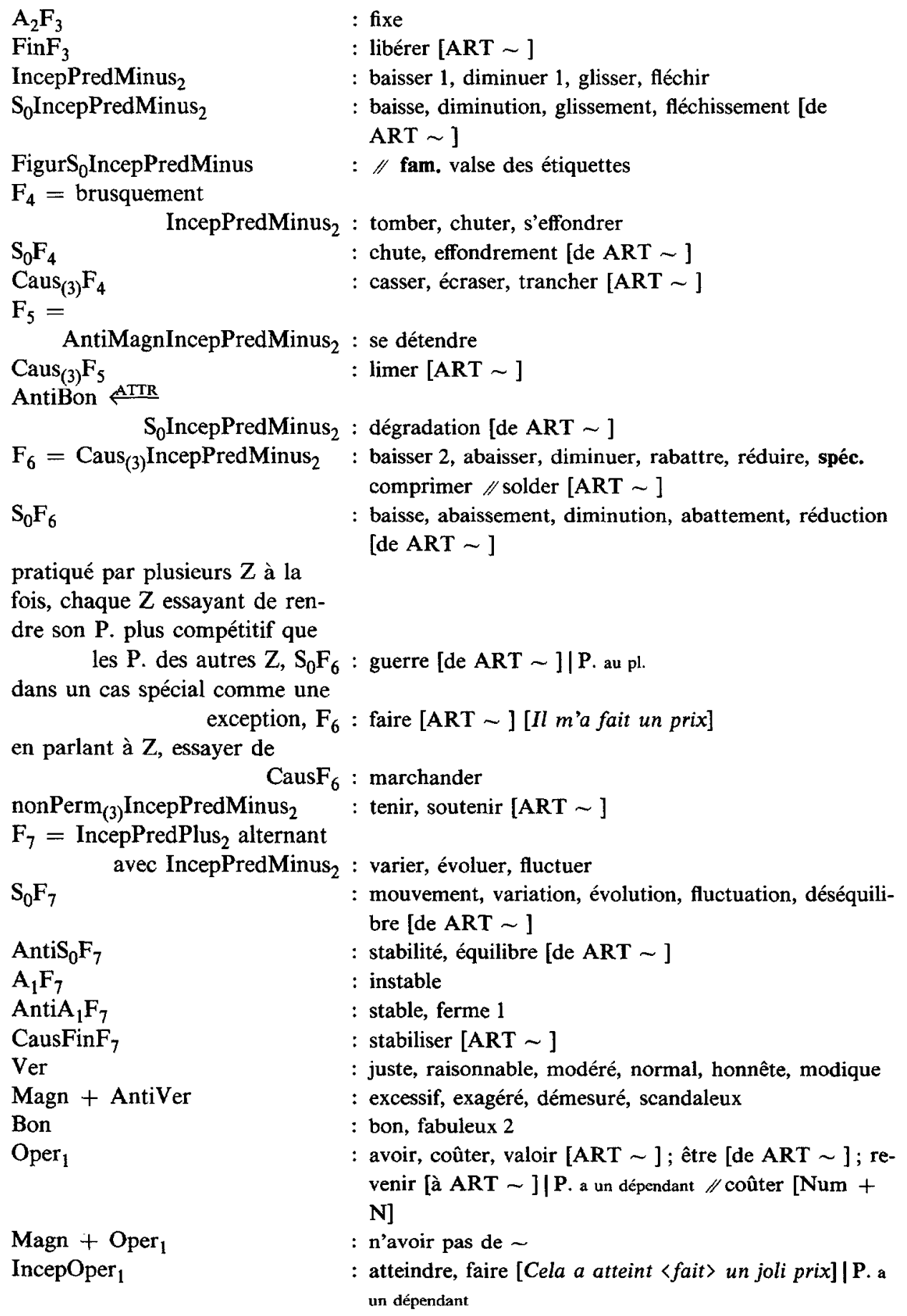




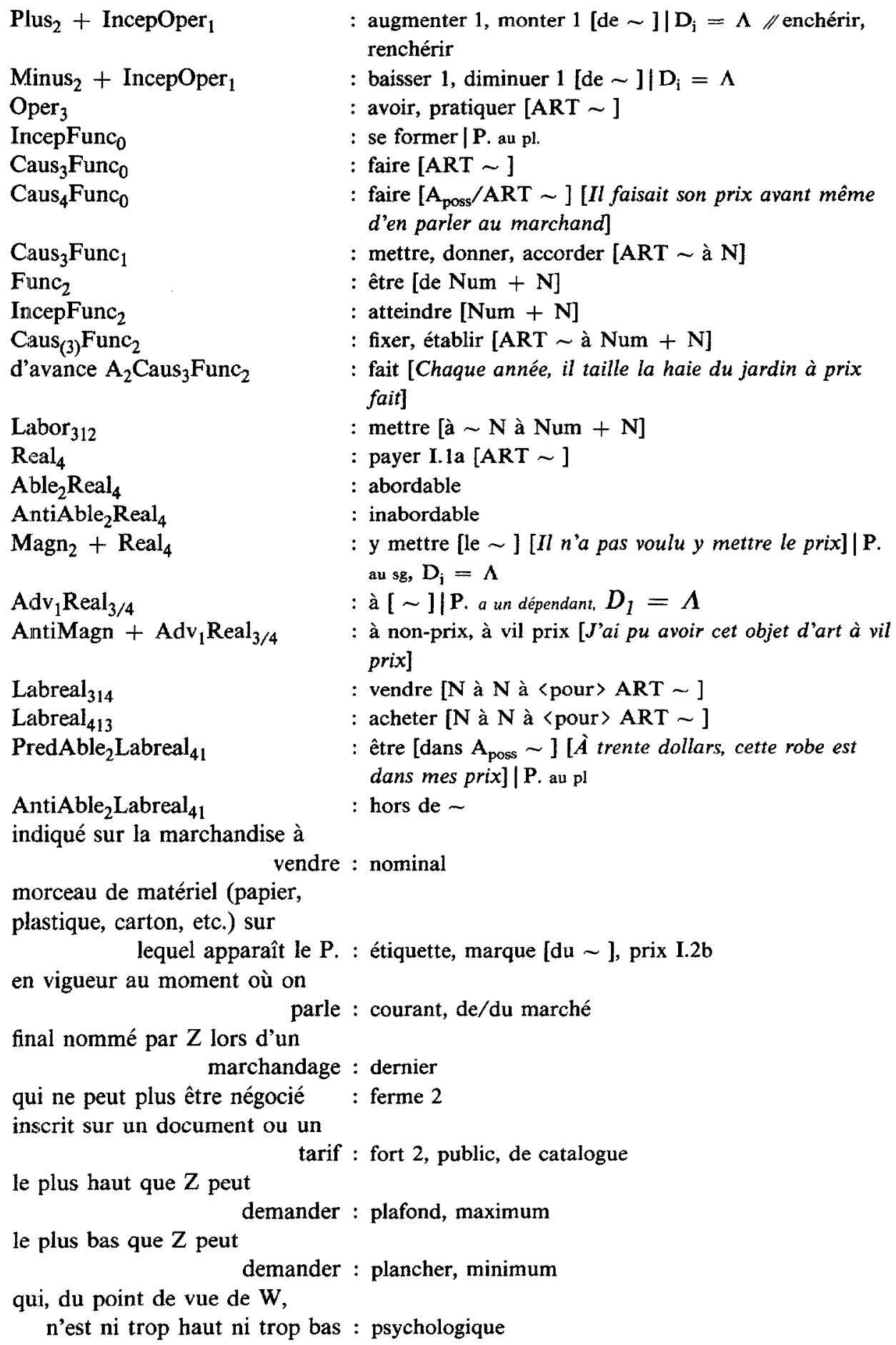


établi pour des raisons politiques, sociales, nationales, éthiques, religieuses, etc. : politique par unité de $\mathrm{X}$ : unitaire, à l'unité, à la pièce

auquel $\mathrm{X}$ est vendu par $\mathrm{Z}$ -

grossiste à W-détaillant ou gros consommateur : de gros, de semi-gros

auquel $\mathrm{X}$ est vendu à W-

consommateur final : de détail, au consommateur, à la consommation

$F_{8}$ tel qui ne comprend pas les

taxes dues par $\mathrm{Z}$ à l'occasion de

la vente de $\mathrm{X}$ : hors taxes (HT)

AntiF $_{8}$

: toutes taxes comprises (TTC)

ce que $X$ coûte à $Z$

: coûtant, de revient

tel que $\mathrm{X}$ a à la sortie du maga-

sin commercial du producteur : à la production, du producteur (fabricant), de départ de chez le producteur (fabricant), d'usine, départ dépôt (entrepôt, usine)

préconisé pour $\mathrm{X}$ par le producteur de $\mathrm{X}$ à $\mathrm{Z}$ : conseillé

communiquer le $P$. à $W$

$:$ dire, nommer, donner, accorder [ART $\sim$ à N]

communiquer le $P$. par écrit

$:$ afficher, marquer $[$ ART ]

(de $\mathrm{W})$ communiquer à $Z$ le $P$.

que $\mathrm{W}$ est prêt à payer pour $\mathrm{X}:$ offrir $[\mathrm{ART} \sim$ à N]

\section{Exemples}

Les femmes de ménage ont augmenté leur prix de vingt centimes l'heure pendant les vacances [Céline]. Les prix ont bien monté un peu, mais au prix que tout coûte maintenant, ça ne change pas [Aymé]. Une œuvre où il y a des théories est comme un objet sur lequel on a laissé la marque du prix [Proust]. Elle avait passé par ses conditions en lui cédant la ferme de Presle à un prix dérisoire [Flaubert]. Alors il lâchait brusquement son " dernier prix " qu'il tenait en réserve depuis le matin et $M$. Roch, agréablement surpris, tirait de sa poche un carnet d'ordres [Maurois]. Les vivres étaient rares et hors de prix [France]. Si on me procure le moyen de baisser mes prix de revient, c'est-à-dire de diminuer mes charges fiscales et de réduire les salaires, je serai parfaitement satisfait [Nizan]. Les œuvres de Rembrandt exposées n'ont pas de prix. Le commissaire-priseur a mis à prix cette commode Louis XVI à trois mille francs. Le type qui voudra s'offrir ça, il faudra sûrement qu'il y mette le prix.

1.2b. Étiquette destinée à figurer sur la marchandise en indiquant le prix I.2a de cette marchandise.

\section{Exemples}

Il m'a offert un cadeau, le prix était encore dessus. Il y a eu un coup de vent et tous les prix sont tombés. N'oubliez pas de coller le prix sur les nouveaux articles que nous avons reçus. 
I.3. pas de pl. Prix $X$ de $Y$ [payé par $Z]=X$ dont $Z$ paye III. $1^{*} \mathrm{Y}-$ comme si $X$ était le prix I.2a de $\mathrm{Y}$ exigé de $\mathrm{Z}\left[=\mathrm{S}_{3}\right.$ (payer $\left.\left.I I I .1\right)\right]$.

\section{Régime}

\begin{tabular}{|l|l|l|}
\hline \multicolumn{1}{|c|}{$1=\mathrm{X}$} & \multicolumn{1}{|c|}{$2=\mathrm{Y}$} & $3=\mathrm{Z}$ \\
\hline 1. de $\mathrm{N}$ & 1. de $\mathrm{N}$ & \\
\hline
\end{tabular}

1) $\mathrm{C}_{1}$

2) $\mathrm{C}_{1}+\mathrm{C}_{2}$

$\mathrm{C}_{1}$

$\mathrm{C}_{2}$

Impossible
: ne s'emploie qu'avec l'expression au prix de

: impossible

: au prix d'un long travail

: le prix du succès

: *le prix du succès d'un long travail... (2) [le prix du succès qui était un long travail]

\section{Fonctions lexicales}

Magn

Ver

Oper $_{1}$

: haut, élevé, d'or

: juste/prépos

: être [ART ]

: coûter, valoir $[$ ART $\sim$ ]|P. a un dépendant

: être [N]

: payer [ART ]

: y mettre $[\mathrm{le} \sim]\left[J^{\prime} y\right.$ mettrai le prix $] \mid \mathrm{D}_{\mathrm{i}}=\Lambda$

$:$ à $[\mathbf{A R T} \sim] \mid \mathbf{P}$. a un dépendant, $\mathbf{D}_{1}=\Lambda$

$:$ à tout $\sim[$ Il faut le battre à tout prix $]$ G n'est pas négatif

: litt. payer III.1 [N de $\mathrm{ART} \sim]$; acheter $[\mathrm{N}$ à ART $\sim] \mid$ P. a un dépendant

\section{Exemples}

Il y a quelque chose de trouble que je veux éclaircir à tout prix [Gide]. Le silence, l'étouffement, je ne connais que ça; j'agirai, j'y mettrai le prix [Mauriac]. Il y a un prix à payer pour toute chose. Elle fait instruire son fils au prix de lourds sacrifices. Il a acheté la. victoire au prix de sa vie.

* $X$ paie III.I Y de $\mathrm{Z}=$ Personne $\mathrm{X}$ perd $\mathrm{Z}$ comme résultat de ses efforts visant la réalisation de $\mathrm{Y}$. 
I.4. pas de pl. Prix $[X]$ de $Y[$ payé par $Z]=\mathrm{X}$ dont $\mathrm{Z}$ paye $\mathrm{III} .2^{*} \mathrm{Y}$ - comme si $\mathrm{X}$ était le prix I.2a exigé de $Z\left[=S_{3}\right.$ (payer III.2)].

\section{Régime}

\begin{tabular}{|c|l|l|}
\hline $1=\mathrm{X}$ & \multicolumn{1}{|c|}{$2=\mathrm{Y}$} & $3=\mathrm{Z}$ \\
\hline & 1. de $\mathrm{N}$ & \\
\hline
\end{tabular}

$\mathrm{C}_{2}$

: le prix d'une mauvaise action

\section{Fonctions lexicales}

Ver

Oper $_{1}$

$:$ juste

Oper $_{2}$

: être [ART $\sim$ ] [La mort sera le prix de ses forfaits]

: coûter $[\mathrm{ART} \sim$ ] [Imaginez le prix que cela va lui coûter]

Func $_{1}$

$\mathrm{Real}_{3}$

: être [N] [Le prix de ses abus a été la maladie]

: payer $[\mathrm{ART} \sim$ ]

\section{Exemples}

Il devra payer le prix de sa faute. Une amende de $30 \$$ est le prix d'une infraction au code de la route. La mort des premiers nés a été le prix de l'entêtement du Pharaon.

I.5. Prix $[X]$ de $Y[$ payé par $\mathrm{Z} a \grave{ } W]=\mathrm{X}$ dont $\mathrm{Z}$ paye II.a** $\mathrm{W}$ pour une action $\mathrm{Y}$ de $\mathrm{W}$ - comme si X était le prix I.2a de $\mathrm{Y}\left[=\mathrm{S}_{3}\right.$ (payer II.a)].

\section{Régime}

\begin{tabular}{|c|l|c|c|}
\hline $1=\mathrm{X}$ & \multicolumn{1}{|c|}{$2=\mathrm{Y}$} & $3=\mathrm{Z}$ & $4=\mathrm{W}$ \\
\hline & $\begin{array}{l}\text { 1. } d e \mathrm{~N} \\
\text { 2. } d e \mathrm{~V}_{\mathrm{inr}} \\
\text { 3. pour } \mathrm{V}_{\mathrm{inf}}\end{array}$ & - & - \\
\hline
\end{tabular}

1) $\mathrm{C}_{2.2}$

: ne s'emploie qu'avec l'expression pour prix

$\mathrm{C}_{2}$

: le prix du dévouement / de l'avoir aidé / pour l'avoir aidé

\section{Fonctions lexicales}

$\operatorname{Syn}_{n}$

: récompense

* X paye III.2 Y de $\mathrm{Z}$ à $W=$ Personne $\mathrm{X}$ subit, par suite de son action $\mathrm{Y}$ (désagréable pour $\mathrm{W}$ ), des conséquences $Z$ désagréables pour $X$ (et provoquées par $W$ ) - comme si $X$ payait I.la $Z$ à $W$ pour $Y$.

** $X$ paye $Y$ de $Z$ pour $W=X$ traite $Y$ de façon $Z$ en réponse à l'action $W$ faite par $Y, Z$ étant considéré comme équivalent de $\mathrm{W}$ - comme si $\mathrm{X}$ payait I. la $\mathrm{Z}$ à $\mathrm{Y}$ pour $\mathrm{W}$. 


Magn
AntiMagn
Adv 1
Oper $_{1}$
Oper $_{3}$
Oper $_{4}$
quel que soit le P., Adv Oper $_{4}$
Func

Magn

AntiMagn

: haut, élevé

bas, faible, dérisoire, vil

: pour [ ] [Pour prix d'avoir travaillé, il n'a reçu que des remontrances] $\mid \mathrm{D}_{2} \neq \Lambda, \mathrm{D}_{2} \neq \mathrm{C}_{2.3}$

: être $[\mathrm{ART} \sim$ ] [Un baiser fut le prix de sa galanterie]

: payer $[\mathrm{ART} \sim$ ]

: recevoir $[\mathrm{ART} \sim]$

: à aucun $\sim$ [Je ne le lui donnerai à aucun prix] $\mid \mathrm{G}$ est négatif

: être [N] [Le prix de sa sagesse était une crème glacée]

\section{Exemples}

Je le marie, et pour prix d'avoir eu par mes soins son épouse, il veut intercepter la mienne [Beaumarchais]. Il était serviable, empressé même, mais pas très adroit, de sorte que, pour prix de ses soins, il recevait moins de remerciements que de rebuffades [Gide]. Les legs qu'il reçut étaient le prix d'un long dévouement.

Il.1 Prix de $X$ de $Y$ de $Z$ pour $W$ de $U$ de $V=$ Récompense $X$ offerte par $Y$ dans le cadre d'un concours $\mathrm{V}$ dans un domaine $\mathrm{U}$ à la personne $\mathrm{Z}$ dont la réalisation $\mathrm{W}$ dans $\mathrm{U}$ sera considérée comme la meilleure.

\section{Régime}

\begin{tabular}{|c|l|l|l|l|l|}
\hline $1=\mathrm{X}$ & \multicolumn{1}{|c|}{$2=\mathrm{Y}$} & \multicolumn{1}{|c|}{$3=\mathrm{Z}$} & \multicolumn{1}{c|}{$4=\mathrm{W}$} & \multicolumn{1}{c|}{$5=\mathrm{U}$} & \multicolumn{1}{c|}{$6=\mathrm{V}$} \\
\hline 1. de $\mathrm{N}_{\text {num }}$ & $\begin{array}{l}\text { 1. de } \mathrm{N} \\
\text { 2. } \mathrm{A}\end{array}$ & $\begin{array}{l}\text { 1. de } \mathrm{N} \\
\text { 2. } \mathrm{A}_{\text {poss }}\end{array}$ & 1. pour $\mathrm{N}$ & $\begin{array}{l}\text { 1. de } \mathrm{N} \\
\text { 2. } \mathrm{A}\end{array}$ & $\begin{array}{l}\text { 1. de } \mathrm{N} \\
\text { 2. } \mathrm{A}\end{array}$ \\
\hline
\end{tabular}

1) $\mathrm{C}_{2.2}$

2) $\mathrm{C}_{5.2}$

3) $C_{i}+C_{j}+C_{k}$

4) $C_{i}+C_{j}$

5) $\mathrm{C}_{1}+\mathrm{C}_{2}+\mathrm{C}_{3}+\mathrm{C}_{4}+$

$\mathrm{C}_{5}+\mathrm{C}_{6}:$ impossible

$\mathrm{A}=$ académique, scolaire,...

: $\mathbf{A}=$ littéraire, scientifique,...

: impossible $\mid \mathrm{C}=$ de $\mathrm{N}$

: impossible $\mid \mathrm{C}=\mathrm{A}$

$\mathrm{C}_{1}+\mathrm{C}_{2}$

$\mathrm{C}_{1}+\mathrm{C}_{2}+\mathrm{C}_{3}$

$\mathrm{C}_{1}+\mathrm{C}_{2}+\mathrm{C}_{3}+\mathrm{C}_{4}$

$\mathrm{C}_{1}+\mathrm{C}_{3}+\mathrm{C}_{4}+\mathrm{C}_{5}$

$\mathrm{C}_{1}+\mathrm{C}_{3}+\mathrm{C}_{4}+\mathrm{C}_{6}$

$\mathrm{C}_{2}+\mathrm{C}_{3}+\mathrm{C}_{4}+\mathrm{C}_{5}$
: un prix de $1000 \$$ de l'Académie / académique

: le prix de Pierre / son prix académique de $1000 \$$

: son prix académique de $1000 \$$ pour le meilleur disque

: son prix littéraire de $500 \$$ pour le meilleur roman

: son prix olympique de $5000 \$$ pour le saut le plus haut

: son prix scolaire de sciences pour ses résultats 
Impossible

Syn $_{\supset}$
$\mathrm{S}_{3}$
$\mathrm{~S}_{4 \subset}$
$\mathrm{A}_{1}$
$\mathrm{Ver}$
$\mathrm{Caus}_{2} \mathrm{Oper}_{1}$
Conv $_{3214} \mathrm{Caus}_{2}$ Oper $_{1}$
Oper $_{2}$
$\mathrm{~F}_{1}=$ à plusieurs Z, Oper
cérémonie à l'école durant

\author{
: *un prix académique littéraire $[=$ un prix \\ académique de littérature] \\ * un prix de $100 \$$ des jeux olympiques du plus \\ haut saut $[=$ un prix olympique de $100 \$$ \\ pour le saut le plus haut]
}

\section{Fonctions lexicales}

: récompense

: prix II.2b ; récipiendaire, lauréat, gagnant [de ART ]

: prix II.2a

: de $\sim$ [un livre de prix]

: mérité

: donner [ $\mathrm{N}$ à $\mathrm{N}$ en $\sim$ ] [On lui a donné un livre en prix]

: recevoir $[\mathbf{N}$ de $\mathbf{N}$ en $\sim]$

: donner, décerner, offrir, remettre [ART à N]

distribuer [ART à N]

laquelle $\mathrm{F}_{1}$ : distribution [des $\sim \mathrm{s}$ ]

\section{Oper $_{3}$}

: avoir, obtenir, recevoir, gagner, emporter, remporter ; mériter ; fam. décrocher [ART ]

\section{essayer $\mathrm{Oper}_{3}$ \\ Caus $_{(2)}$ Func $_{0}$ \\ Funcactual \\ Funcusual \\ Caus $_{4}$ Func $_{3}$}

tous les P. Oper

: fam. raffler $[$ ART $\sim] \mid P$. au pl

$\mathrm{S}_{1}$ (essayer $\mathrm{Oper}_{3}$ )

: disputer $[\mathrm{ART} \sim$ ]; se battre [pour ART ]

: aspirant $[\mathbf{a ̀ ~ A R T ~}$ ]

pendant un certain temps $\mathrm{Oper}_{3}:$ détenir [ART $\sim$ ]

plusieurs fois de suite Oper $_{3} \quad$ : conserver [ART $\sim$ ]

sans y avoir droit Oper

: s'arroger, s'approprier [ART ]

: créer, fonder, établir, instituer [ART ]

: aller [à $\mathbf{N}$ ]

: récompenser $[\mathrm{N}]$

: mériter $[\mathrm{ART} \sim$ à N] [Sa sculpture lui a mérité un prix]

décider qu'il faut que Funcactual

Caus $_{2}$ FinFunc $_{3}$

Labor $_{23}$

accorder, adjuger, attribuer $[\mathrm{ART} \sim$ à N]

: retirer $[\mathrm{ART} \sim \mathrm{de} \mathrm{N}]$

: couronner $[\mathrm{N}$ de $\mathrm{ART} \sim]$

le P. le plus important dans

certains concours : grand

donné à ceux qui n'ont pas ga-

gné un des $\mathbf{P}$. importants mais

qu'on veut encourager : de consolation 


\section{Les différents prix du monde}

Prix Nobel

Prix Goncourt

Prix Lénine

Prix Femina

Prix Pulitzer

: fondé par Alfred Nobel, savant suédois, et destiné à récompenser les bienfaiteurs de l'humanité dans cinq domaines différents : physique, chimie, médecine, littérature, actes sociaux ou politiques remarquables (Suède).

: porte le nom de deux frères écrivains et décerné à la fin de chaque année, par l'Académie des Goncourt, au meilleur roman, au meilleur recueil de nouvelles, au meilleur volume d'imagination en prose (France).

: fondé par le gouvernement soviétique et destiné à récompenser des savants, artistes et écrivains d'U.R.S.S. (U.R.S.S.)

: décerné en fin d'année, quelques jours avant le prix Goncourt, à une ouvre d'imagination (France).

: institué par Joseph Pulitzer, journaliste américain, et décerné chaque année par l'université Columbia à des représentants des différents domaines du journalisme (U.S.A.).

Prix Théophraste-Renaudot : fondé par un groupe d'informateurs littéraires et décerné chaque année à l'auteur d'un roman, d'un récit ou d'un recueil de contes ou de nouvelles. Ne comporte pas de prix en argent (France).

Prix distribués aux écoliers dans le système scolaire français

Prix d'excellence

Prix d'honneur
: offert en fin d'année à l'élève qui a obtenu de très bons résultats dans toutes les matières étudiées.

: inférieur au prix d'excellence et offert aux élèves qui ont obtenu de bons résultats.

\section{Exemples}

Au lieu de faire un prix Monthyon pour la récompence de la vertu... [Gauthier]. Philibert avait remporté tous les prix au Collège ; le fait est qu'en sortant il ne savait rien [Stendhal]. Elle remporte partout le prix de beauté. François Nourissier a obtenu le grand prix du roman de l'Académie française. La distribution des prix marque la fin de l'année scolaire. Il avait une étagère garnie des prix qu'il avait gagnés dans des compétitions sportives. Il a reçu six beaux prix à reliure rouge et dorés sur tranche.

II.2a. Prix $[X]$ de $Y$ de $Z=$ Oeuvre $X$ exécuté dans le domaine $Z$ pour lequel l'auteur de $\mathrm{X}$ a reçu un prix $\mathrm{II} .1$ décerné par $\mathrm{Y}\left[=\mathrm{S}_{4}(\right.$ prix $\left.I I .1)\right]$. 


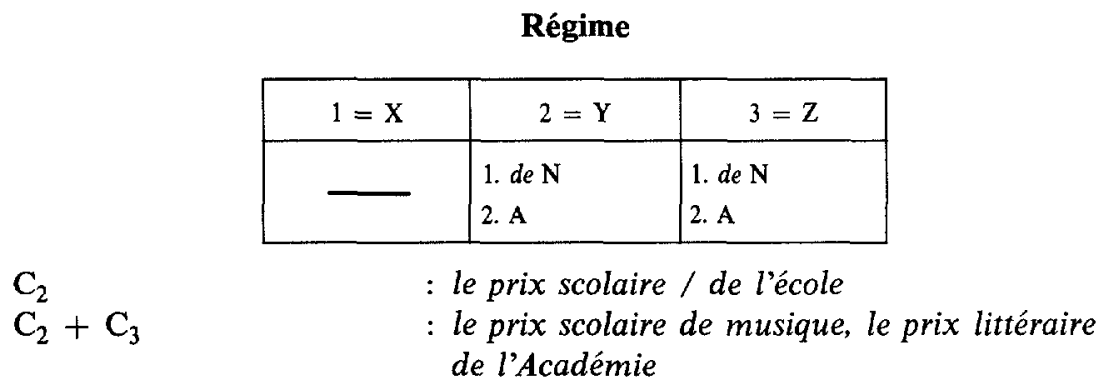

\section{Exemples}

Lisez le dernier prix du roman de l'Académie française. Un prix Goncourt doit-il être un livre populaire ou un livre difficile?

II.2b. Prix $[X]$ de $Y$ de $Z=$ Personne $\mathrm{X}$ qui a reçu un prix II.1 décerné dans le domaine $\mathrm{Z}$ par $\mathrm{Y}\left[=\mathrm{S}_{3}(\right.$ prix II. $\left.)\right]$.

\section{Régime}

\begin{tabular}{|c|l|l|}
\hline $1=\mathrm{X}$ & \multicolumn{1}{|c|}{$2=\mathrm{Y}$} & \multicolumn{1}{|c|}{$3=\mathrm{Z}$} \\
\hline & 1. de $\mathrm{N}$ & 1. de $\mathrm{N}$ \\
\hline
\end{tabular}

$\mathrm{C}_{2}$
$\mathrm{C}_{2}+\mathrm{C}_{3}$

: prix du Conservatoire

: prix de musique du Conservatoire

\section{Exemples}

Un professeur de biologie ou de physiologie, fût-il prix Nobel, ne peut pas enseigner à la faculté de médecine. C'est un ancien prix d'excellence. Elle a épousé un prix de musique. Oui, répondit-il, j'ai joué pas mal dans le temps, j'ai été premier prix du Conservatoire de 19... Il y avait deux prix Goncourt qui accompagnaient le ministre des Affaires culturelles.

BetTy COHEN et Léo ElNitsky 